\title{
Barreras geográficas: ¿̇lntervienen en la composición y distribución longitudinal en la comunidad íctica de un río?
}

\section{Geographic barriers: Do the intervene in the composition and longitudinal distribution of the fish community in a river?}

\section{Sam Catchpole ${ }^{1,2, *}$, Víctor Rojas ${ }^{1}$, Marisel Medina ${ }^{1} \&$ Víctor Hugo Ruiz $^{3}$}

${ }^{1}$ Fundación Organización para el Desarrollo y la Sustentabilidad (ODyS), Concepción, Región del Biobío, Chile.

2Pares \& Álvarez Gestión Ambiental, Concepción, Región del Biobío, Chile.

${ }^{3}$ Departamento de Zoología, Facultad de Ciencias Naturales y Oceanográficas, Universidad de Concepción, Casilla 160-C, Concepción, Región del Biobío, Chile.

*Email: scatchpole1984@gmail.com

\section{RESUMEN}

Diversas son las variables que intervienen en la distribución y ocupación espacio-temporal de peces en un sistema fluvial. En este estudio evaluamos variables geográficas (cascadas, saltos, rápidos, pendiente, altitud) y fisicoquímicas (velocidad de corriente, $\mathrm{pH}$, conductividad y oxígeno disuelto) en distintos tramos del río Quimán y su relación con el ensamble de peces. Este río, al ser cordillerano, presenta una orografía abrupta con diversas cascadas, saltos y rápidos que afectan su conectividad. Por ello, la hipótesis de trabajo fue establecer si estas variables geográficas y fisicoquímicas pueden influir en la segregación espacial de los peces en el río. Los resultados mostraron una variación significativa (ANOSIM $r=0,6 p=0,0006$ ) en la composición del ensamble a lo largo del río, definiendo una segregación espacial por parte de las especies nativas, y un uso de hábitat preferencial por parte de las especies nativas en ambientes de baja velocidad de corriente $(<0,64$ $\mathrm{m} \mathrm{s}^{-1}$ ) y pendientes menores al 3,5\%. Sin embargo, mediante el análisis BEST/BIOENV determinó una baja relación entre las variables ambientales y la distribución del ensamble de peces $(r=0,37 p=0,03)$ en el Río Quimán. Por otra parte, la frecuencia de cascadas, saltos y rápidos disminuyeron en el sentido de la corriente, en contraste con la riqueza y diversidad, aun cuando existe una piscicultura y una planta de tratamiento en el tramo terminal. Se sugiere desarrollar estudios más específicos para entender la influencia que ejercen las variables geográficas sobre las poblaciones de peces nativas.

Palabras claves: barreras naturales, peces nativos, salmonídeos, variación espacial.

\section{ABSTRACT}

Several variables are involved in the patterns of distribution and spatio-temporal occupation of fish in a fluvial system. In this study, we evaluate numerous geographic (cascades, waterfalls and riffles) and physicochemical variables (slope, current velocity, pH, conductivity and dissolved oxygen) in different sections of the Quimán River, and its relationship with fish assemblage. This river is a mountain stream and presents an abrupt orography with various waterfalls, cascades and riffles that affect the connectivity of the river. Therefore, the working hypothesis was to establish if some of these geography and physicochemical variables can influence the spatial segregation of fish in the river. The results showed a significant variation (ANOSIM r $=0.6 p=$ 0.0006 ) in the composition of the assemblage along the river, defining a spatial segregation for native species, and a preferential habitat use was established by native species due to low current velocity environments $<$ $0.64 \mathrm{~m} \mathrm{~s}^{-1}$ ) and slope less than 3.5\%. Nevertheless, a BEST/BIOENV analysis determined a low relationship 
between environmental variables and fish assemblage distribution $(r=0.37 p=0.03)$. On the other hand, frequency of waterfalls, cascades and riffles decreased in the direction of the flow, in contrast to richness and diversity, even when there is a pisciculture and treatment plant of wastewater in the terminal section. It is suggested to develop more specific studies to understand the influence of geographic variables on native fish populations.

Keywords: natural barriers, native fish, salmonids, spatial variation.

\section{INTRODUCCIÓN}

Diversas son las variables que influyen en la conectividad, distribución y ocupación espacio-temporal de hábitats por parte de las distintas especies. Para el caso de los peces de aguas continentales, una de las variables más relevantes son las barreras geográficas. Rahel (2007) describe tres escalas de barreras geográficas para los ecosistemas dulceacuícolas: continental, entre cuencas hidrográficas y dentro de las cuencas. Dentro de las cuencas se presentan: cascadas, saltos de agua, rápidos y altas pendientes generan una barrera que restringe la conectividad y ocupación de hábitats para los peces. Para las especies nativas estas barreras geográficas son restrictivas, debido sus pequeños tamaños $(<15 \mathrm{~cm}$ de LT) (Vila \& Habit 2015) y características anatómicas no adaptadas para habitar aguas turbulentas y para el ascenso a pendientes abruptas (Arratia 1982, 1987; Vila et al. 1999). Generando desplazamientos limitados, y ámbitos de hogar y distribuciones latitudinales restringidas para la mayoría de las especies nativas, con ciertas excepciones como los géneros Diplomystes, de amplios desplazamientos pasivos, y Aplochiton de alta movilidad como se postula (Piedra et al. 2012; Victoriano et al. 2012), y la especie Trichomycterus areolatus de amplia distribución latitudinal, habitando desde el río Huasco $\left(28^{\circ} 27^{\prime} \mathrm{S}\right)$ a la Isla de Chiloé $\left(41^{\circ} 27^{\prime} \mathrm{S}\right)$ (Dyer 2000).

En la actualidad, escasos son los estudios que describen las capacidades y modalidades de nado de especies nativas (Link \& Habit 2015), así como los usos, preferencias y disponibilidad de hábitats, a excepción de los estudios de Laborde et al. (2016) con Cheirodon galusdae y Basilichthys microlepidotus, García et al. (2012) con Percilia irwini y García et al. (2011) con Trichomycterus areolatus. No obstante, a pesar de ello, aún no se tiene información concreta sobre la influencia que ejerce una variable geográfica (llámese como rápido, pendiente abrupta, cascada o salto), y cuando esta se considera una barrera natural infranqueable que afecta y modifica la distribución y composición espacial de los ensambles nativos.
En cuanto a los salmonídeos, introducidos a fines de 1800 en Chile con fines comerciales (Basulto 2003), corresponden al grupo de mayor riqueza de especies exóticas (12 especies), donde Salmo trutta (Trucha marrón) y Oncorhynchus mykiss (Trucha arcoíris) se encuentran ampliamente distribuidas en el país (Habit et al. 2015), estableciendo poblaciones asilvestradas que han afectado negativamente a la ictiofauna dulceacuícola nativa (Arismendi et al. 2009, 2012). Este éxito de expansión se debe entre otros factores a sus capacidades de natatorias que les permiten acceder y colonizar nuevos hábitats (Hawkins \& Quinn 1996). Sin embargo, existen limitaciones para estos peces, por ejemplo, Chisholm \& Hubert (1986) determinaron que pendientes sobre un 6,8 \% limitarían movimientos activos de salmónidos hacia tramos fluviales superiores. Mientras que Kruse et al. (1997) describieron la ausencia de salmónidos en pendientes sobre el $10 \%$. Asimismo, la presencia de rápidos, saltos y cascadas naturales en sistemas fluviales afectan los procesos de dispersión y definen diferencias de riqueza y diversidad entre los ensambles de peces localizados antes y después de estas barreras (Robinson \& Rand 2005; Olden et al. 2010; Torrente-Vilara et al. 2011; Silva et al. 2015). También son precursoras de crear nuevos hábitats acuáticos (Rahel 2007, Torrente-Vilara et al. 2011), aumentar la segregación entre los ensambles (Hubert \& Renno 2006; Olden et al. 2010) y de promover procesos de endemismo (Ricklefs \& Schluter 1993).

El Río Quimán es uno de los tantos afluentes del Lago Ranco (sur de Chile) y tiene su origen en los faldeos cordilleranos de la Región de Los Ríos (Campos 1984). Debido sus características montañosas, presenta una marcada variación altitudinal, altas pendientes, aguas turbulentas y numerosas barreras naturales (cascadas, saltos y rápidos) a lo largo de su recorrido (Parot 2012), lo cual podría estar afectando la conectividad y distribución espacial de los peces presentes en la cuenca. Por lo anterior, la hipótesis de trabajo fue determinar si estas variables geográficas (cascadas, saltos, rápidos, pendientes, altitud y velocidad de 
corriente) y fisicoquímicas (temperatura, oxígeno disuelto, $\mathrm{pH}$ y conductividad), limitan o segregan espacialmente la ictiofauna nativa presente en los distintos tramos del Río Quimán.

El objetivo general es establecer potenciales patrones de distribución longitudinal y composición íctica, en función de la geografía (pendientes, rápidos, cascadas y saltos) y las variables fisicoquímicas de la columna de agua a lo largo del Río Quimán.

\section{MATERIALES Y MÉTODOS}

\section{ÁREA DE ESTUDIO}

La cuenca hidrográfica del Río Quimán abarca un área de drenaje de $42,75 \mathrm{~km}^{2}$, ubicada en el lado oriental de la cuenca del Lago Ranco, en la cordillera de Futrono (Huechan 1997). El Quimán chico, su principal tributario, corre paralelo hasta su convergencia en el tramo superior de la cuenca. Actualmente, en esta convergencia se encuentra operando una pequeña central hidroeléctrica de pasada de 2,1 MW desde el año 2015. El régimen de la cuenca es principalmente pluvial, con un aporte nival de solo un $5 \%$ durante el periodo estival (Parot 2012). La geografía del valle del Río Quimán, en su tramo superior, es estrecho y profundo, con riberas abruptas y de exuberante vegetación nativa, mientras que su tramo medio y terminal se abre para formar una planicie, drenando sobre un cajón fluvial hasta su desembocadura en el Lago Ranco. Es en este último tramo del río donde se encuentran actualmente operando una Planta de Tratamiento de Agua Servida (PTAS) y una piscicultura (Fig. 1). La presencia de aguas turbulentas y una alta velocidad de corriente sería el resultado de un alto gradiente del río que favorece una estructura general de rápido-pozón, con presencia de numerosas barreras (cascadas, saltos y rápidos) en su tramo superior y medio. La profundidad máxima determinada para este río fue de 5,0 metros en la zona de bocatoma de la central hidroeléctrica, y de 2,5 metros en la desembocadura del río.

\section{VARIABLES BIOGEOGRÁFICAS Y AMBIENTALES}

Se zonificó longitudinalmente el Río Quimán en tres zonas: alta, media y terminal, de acuerdo sus las características morfológicas del cauce (pendiente, ancho, presencia o ausencia de rápidos, cascadas, saltos y velocidad de corriente). Los rápidos fueron clasificados y definidos como someros y profundos según los criterios establecidos por García et al. 2012. El salto fue definido con un fuerte desnivel donde el flujo de agua cae verticalmente $\left(90^{\circ}\right)$, mientras que la cascada es un desnivel escalonado, donde el flujo de agua cae según la pendiente con desnivel $\left(<90^{\circ}\right)$. En cada zona fluvial se desarrolló en triplicado estaciones de medición de los parámetros fisicoquímicos: oxígeno disuelto ( $\left.\mathrm{mg} \mathrm{L}^{-1}\right)$, conductividad $\left(\mu \mathrm{S} \mathrm{cm}{ }^{-1}\right)$, temperatura $\left({ }^{\circ} \mathrm{C}\right)$, $\mathrm{pH}$ y ORP ( $\mathrm{mV} \mathrm{L}^{-1}$ ), y las variables geográficas: pendiente (\%), velocidad de corriente superficial $\left(\mathrm{m} \mathrm{s}^{-1}\right)$, altitud (msnm), frecuencia y altura de cascadas y saltos (m) (Tabla 1). Los datos de pendiente fueron medidos mediante un clinómetro en transectos de 100 metros. Las cascadas y saltos fueron medidas con un distanciómetro laser Bosh modelo GLM 20. Cada barrera geográfica (cascada, salto y caída) fue contabilizada y referenciada mediante un GPS Garmin Montana 650. La altitud fue obtenida de cartas del Instituto Geográfico Militar (IGM) escala 1:50.000 y corregidas por GPS. La velocidad de corriente fue determinada mediante un flujómetro, marca General Oceanics, modelo 2030 CF en tres transectos perpendiculares a distintas profundidades (superficial, medio y fondo) para cada punto de muestreo.

\section{Muestreo de PeCES}

Doce estaciones de muestreo fueron muestreadas a lo largo del río (cuatro en la zona alta del río, cinco en la zona media y tres en la zona terminal) en otoño, invierno y primavera, durante los años 2016 - 2017 (Fig. 1). En cada estación de muestreo, se recorrió un transecto de 100 metros de largo mediante un equipo portátil de pesca eléctrica SAMUS 725 MP (200-650 Watts de potencia), en un periodo de tiempo de 40 minutos para estandarizar las capturas. Para los hábitats de mayor profundidad (> 1,50 m) se utilizaron redes de monofilamento de enmalle 10 y $15 \mathrm{~mm}$ (dimensiones 2,5 $\mathrm{m}$ de alto $\times 10 \mathrm{~m}$ de largo) en un área de muestreo de 50 $\mathrm{m}^{2}$ durante un tiempo de exposición de 6 horas por sitio de muestreo. Los peces capturados fueron sedados con Benzocaína al $20 \%$ (BZ-20) y mantenidos vivos en recipientes aireados e identificados in situ hasta nivel de especie. A cada ejemplar se le midió la longitud total (0,1 mm de precisión) y el peso total (0,01 g de precisión). Posteriormente, cada ejemplar fue devuelto en el mismo lugar de captura.

\section{ANÁLISIS ESTADÍSTICOS}

- VARIABles AMBientales: ANOVA y ACP

Para determinar diferencias entre las variables geográficas y fisicoquímicas en las distintas zonas fluviales, se utilizó un Análisis de Varianza de una vía (ANOVA). Posteriormente, debido a la presencia diferencias significativas entre las zonas fluviales, se aplicó una prueba de comparación múltiple de Tukey (Kruse et al. 1997). Las variables fueron transformadas a logaritmo, excepto el $\mathrm{pH}$, para cumplir los requerimientos de normalidad (prueba de Shapiro-Wilk) y homocedasticidad (prueba de Levene). Siguiendo la metodología y análisis de 
Krebs (1989), correlaciones de Pearson fueron establecidas para determinar relaciones entre las variables geográficas: altitud, pendiente, velocidad de corriente y número de barreras naturales.

Posteriormente, se efectuó un Análisis de Componentes Principales (ACP) entre las variables fisicoquímicas y geográficas para reducir la dimensión de análisis (el conjunto de variables) a los tres componentes principales que describan el mayor porcentaje de variación total de las variables ambientales (Torrente-Vilara et al. 2011). Los datos fueron transformados $\left(\log _{10}\right)$ y normalizados para homogenizar la varianza y minimizar el error de las unidades de medida de las distintas variables (Clarke et al. 2005).

\section{- VAriables ambientales: BarReras geográficas}

Las barreras geográficas contabilizadas y georreferenciadas fueron cartografiadas, y estimadas su altura promedio y frecuencia porcentual para cada zona fluvial.

\section{- DIVERSIDAD Y COMPOSICIÓN DE PECES}

La diversidad de peces fue evaluada a través del índice de Menhinick, cuya fórmula es, donde $S=$ es el número de especies y $\mathrm{N}=$ el número total de individuos. Para detectar posibles diferencias en la diversidad íctica a lo largo de las distintas zonas del río, se aplicó la prueba no paramétrica $U$ de Mann-Whitney.
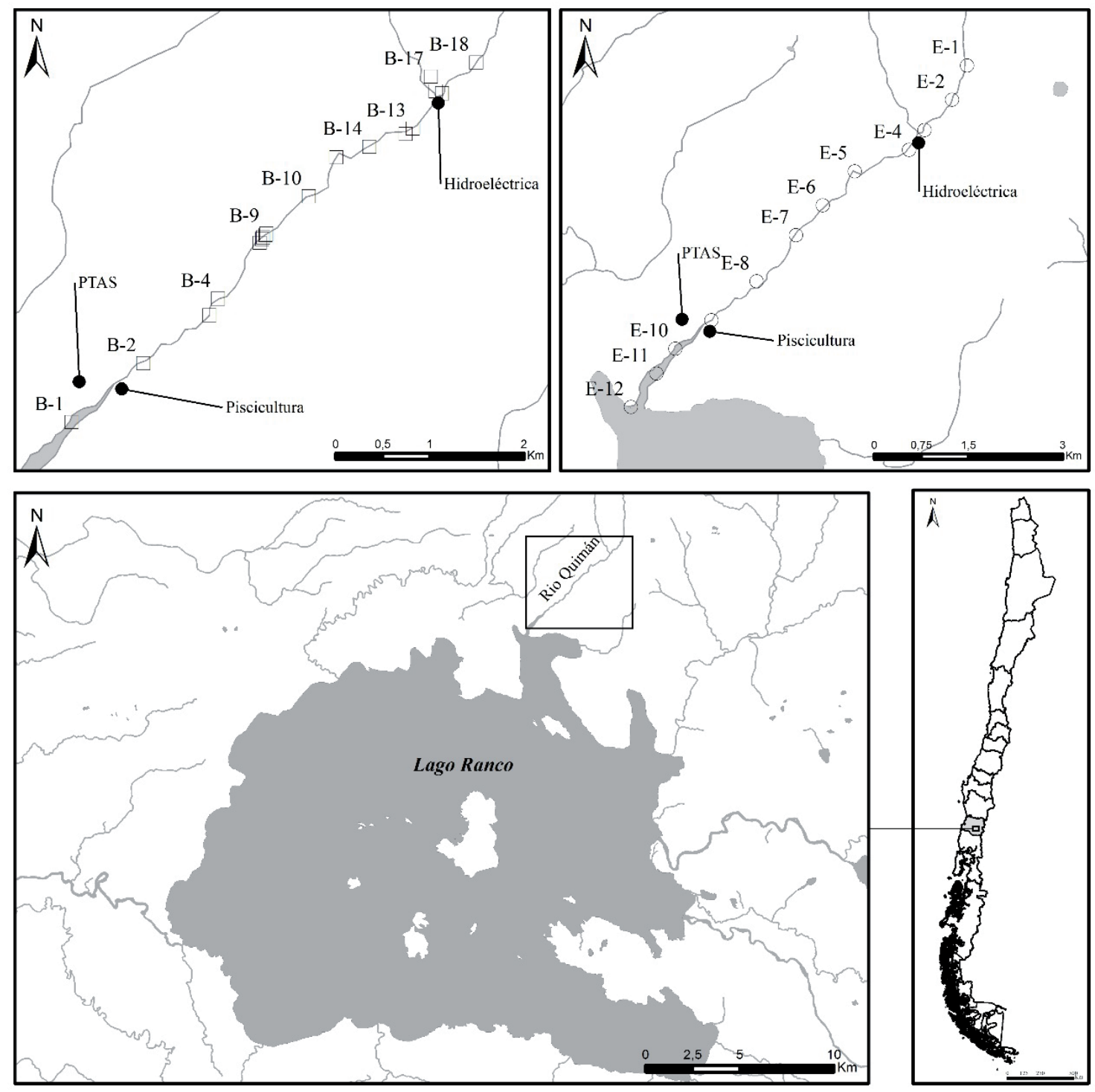

Figura 1. Ubicación de la zona de estudio, las estaciones de muestreo (círculos) y barreras naturales (cuadrados). / Location of the study area, sampling sites (circles) and natural barriers (squares). 


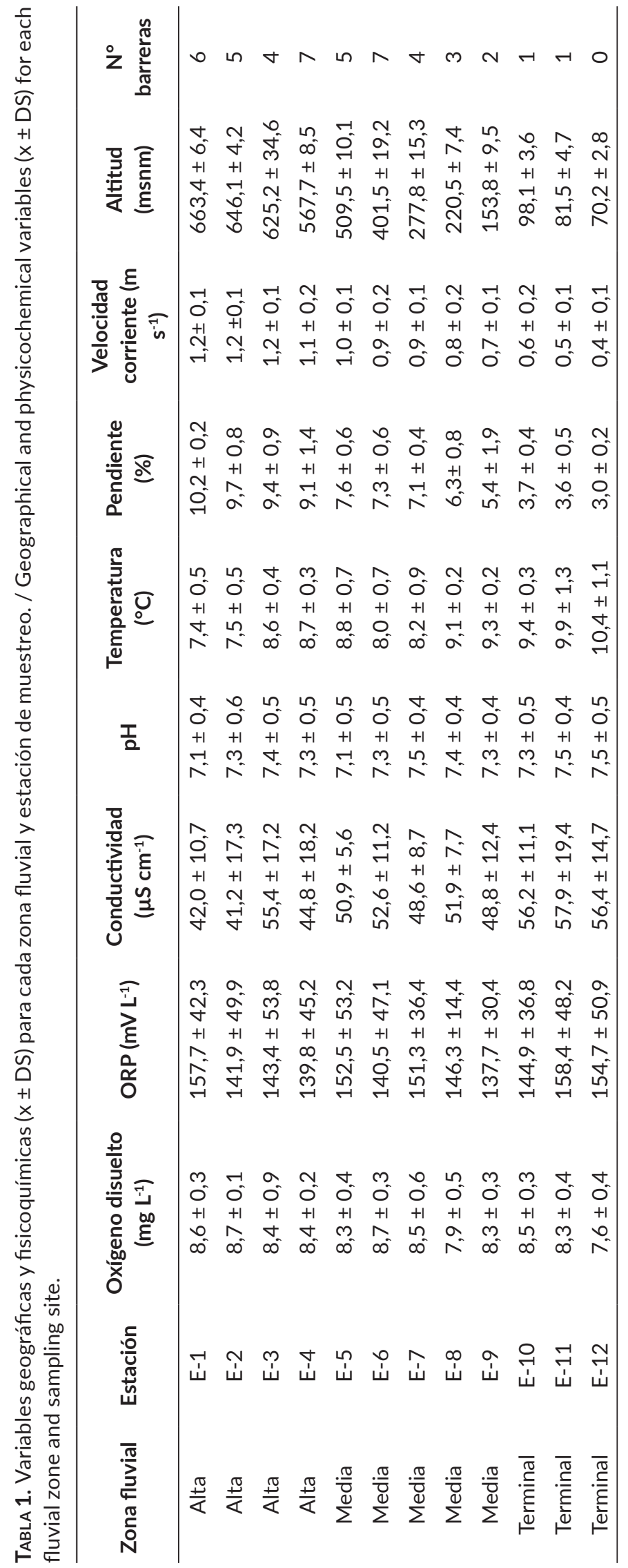

La composición del ensamble de peces a lo largo del río (alta, media y terminal) fue determinada en base a los datos previamente transformadas $(\log x+1)$ de abundancia de especies, mediante la matriz de similitud del índice de BrayCurtis utilizando un análisis de escalamiento multidimensional no métrico NMDS (8 columnas representadas por especies y 12 filas representadas por sitios de muestreo) (Waters et al. 2003) y corroborado mediante un dendrograma de disimilitud de Bray-Curtis, utilizando un algoritmo de grupos pareados (Clarke \& Gorley 2005).

La abundancia fue presentada como captura por unidad de esfuerzo (CPUE) (ind $/ \mathrm{m}^{2} \mathrm{~min}$ ) (Macías-Zamora et al. 1994), y transformada a valores de raíz cuarta para minimizar la influencia de las especies más abundantes e incluir las especies más raras (Ramírez 1999).

Un análisis de similitud (ANOSIM) fue ejecutado para comparar diferencias entre los ensambles de peces distribuidos en las distintas zonas fluviales (Habit et al. 2007). El análisis ANOSIM es una prueba multivariado no paramétrica cuya hipótesis nula determina la igualdad entre dos grupos de muestras (Clarke 1993), mediante una matriz de similitud cuyas distancias han sido convertidas en un rango de valores entre 1 y -1 (Clarke et al. 2005).

Finalmente, para determinar la contribución específica de cada especie en las distintas estaciones y zonas fluviales, se evaluó un análisis porcentual de similitud (SIMPER) en base al índice de similitud Bray-Curtis, mediante el programa PRIMER versión 6.0 (Clarke \& Gorley 2005).

\section{- VARIABLES GEOGRÁFICAS Y ENSAMBLE DE PECES \\ Para determinar una correlación entre las variables geográficas y el ensamble de peces, se utilizó un el análisis BEST/BIOENV (PRIMER versión 6.0). Método no paramétrico que calcula los coeficientes de correlación de Spearman entre matrices de similitud de datos biológicos (basada en la abundancia de especies e índice de Bray-Curtis) y abióticos (basada en variables ambientales y distancia euclidiana) (Clarke \& Warwick 1994).}

\section{RESULTADOS}

\section{VARIABLES AMBIENTALES (GEOGRÁFICAS Y FISICOQUÍMICAS)}

Mediante un análisis de varianza de una vía (ANOVA) y una posterior prueba de comparaciones múltiples de Tukey, se determinó diferencias significativas entre las distintas variables: ORP, $\mathrm{pH}$, altitud, pendiente y velocidad de corriente, en todas zonas fluviales (Tabla 2).

Por otra parte, las correlaciones de Pearson establecieron tendencias directas entre las variables: velocidad de corriente 
y altitud $(r=0,94)$, velocidad de corriente y pendiente $(r=$ $0,91)$ y entre la pendiente y altitud $(r=0,83)$. También, el número de barreras naturales entre la pendiente $(r=0,82)$, velocidad de corriente $(r=0,81)$ y altitud $(r=0,81)$.

Para reducir las variables geográficas y fisicoquímicas se ejecutó un Análisis de Componentes Principales (ACP). Los tres primeros componentes explicaron el 83,6\% de la varianza total de los datos. El primer eje PC1 constituyó el $52,7 \%$ de la varianza y estuvo correlacionado con la velocidad de corriente $(r=-0,44)$, pendiente $(r=-0,43)$ y altitud $(r=$ -0,43). El segundo eje PC2, explicó el 20,6\% de la varianza y se relacionó con el $\mathrm{pH}(r=-0,59)$ y la conductividad $(r=$ -0,53). El tercer eje PC3 determinó el 10,3\% de la varianza total, y correlacionó con el oxígeno disuelto $(r=-0,74)$ y ORP $(r=0,62)$.

En cuanto a las barreras geográficas, se contabilizaron un total de 6 saltos, 7 cascadas y 31 rápidos (23 profundos y 9 someros) (Tabla 3). La altura de los saltos y cascadas fluctuaron entre las distintas zonas fluviales con una media global de $2,4 \pm 0,7 \mathrm{~m}$ y 7,5 \pm 4,4 $\mathrm{m}$, respectivamente. La frecuencia porcentual de las barreras presentó una tendencia general de disminuir progresivamente en el sentido de la corriente, solo identificándose un 3,4\% del total de las barreras contabilizadas en el tramo terminal (Tabla 4).

\section{COMPOSICIÓN DE PECES}

La composición de peces en las 12 estaciones de muestreo, durante los 3 periodos de estudio estuvo constituido por 5 órdenes, 5 familias y 8 especies (6 nativas y 2 introducidas) La riqueza de especies estuvo representada por las familias: Galaxiidae con 3 especies (37,5\%), Salmonidae con 2 especies (25\%), Perciliidae, Atherinopsidae y Trichomycteridae (1 especie), representando cada familia el $12,5 \%$ del total (Tabla 5).

\section{DIVERSIDAD DE PECES POR ZONA FLUVIAL}

La diversidad incrementó en el sentido de la corriente (Fig. 2). Las estaciones E-10, E-11 y E-12 mostraron los mayores índices de diversidad en el Río Quimán. Las diferencias significativas en los ensambles fueron determinadas entre las distintas zonas fluviales: alta/terminal (prueba de MannWhitney $z=-2,178 p=0,029$ ) y media/terminal (prueba de Mann-Whitney $z=-2,087 p=0,036)$. Entre las zonas alta $y$ media no existieron diferencias significativas (prueba MannWhitney $z=-0,435 p=0,628)$. Por otra parte, las mayores abundancias (CPUE) fueron determinadas en la zona terminal, mostrando la misma tendencia que los parámetros riqueza específica y diversidad (Fig. 3).

TABLA 2. Análisis comparativos (ANOVA y posterior prueba de comparaciones múltiples de Tukey) entre las variables geográficas y fisicoquímicas, y los distintos tramos fluviales. / Comparative analyses (ANOVA and post hoc test of Tukey's multiple comparisons) between the geographic and physicochemical variables, and the different river zones.

\begin{tabular}{|c|c|c|c|c|}
\hline \multirow{2}{*}{ Variable } & \multicolumn{2}{|c|}{ ANOVA } & \multicolumn{2}{|c|}{ Prueba Tukey } \\
\hline & Estadístico F & Valor-p & Tramo fluvial & Valor-p \\
\hline \multirow{3}{*}{ ORP } & \multirow{3}{*}{48,77} & \multirow{3}{*}{0,0001} & Alta/Media & 0,0017 \\
\hline & & & Alta/Terminal & 0,043 \\
\hline & & & Media/Terminal & 0,0003 \\
\hline \multirow{2}{*}{$\mathrm{pH}$} & \multirow{2}{*}{31,96} & \multirow{2}{*}{0,0004} & Alta/Media & 0,0034 \\
\hline & & & Alta/Terminal & 0,00073 \\
\hline \multirow{3}{*}{ Altitud } & \multirow{3}{*}{41,73} & \multirow{3}{*}{0,00002} & Alta/Media & 0,0027 \\
\hline & & & Alta/Terminal & 0,0001 \\
\hline & & & Media/Terminal & 0,0046 \\
\hline \multirow{3}{*}{ Pendiente } & \multirow{3}{*}{17,11} & \multirow{3}{*}{0,0008} & Alta/Media & 0,043 \\
\hline & & & Alta/Terminal & 0,00078 \\
\hline & & & Media/Terminal & 0,038 \\
\hline \multirow{3}{*}{ Velocidad } & \multirow{3}{*}{41,13} & \multirow{3}{*}{0,00002} & Alta/Media & 0,006 \\
\hline & & & Alta/Terminal & 0,0001 \\
\hline & & & Media/Terminal & 0,002 \\
\hline
\end{tabular}


TABLA 3. Barreras biogeográficas identificadas por estación de muestreo y zona fluvial. / Biogeographical barriers identified by sampling sites and fluvial zone.

\begin{tabular}{|c|c|c|c|c|c|c|c|}
\hline \multirow{3}{*}{ Zona fluvial } & \multirow{3}{*}{ Estación } & \multicolumn{6}{|c|}{ Barreras biogeográficas } \\
\hline & & \multicolumn{2}{|c|}{ Saltos } & \multicolumn{2}{|c|}{ Cascadas } & \multicolumn{2}{|c|}{ Rápidos } \\
\hline & & $\mathbf{N}$ & Altura (m) & $\mathbf{N}$ & Altura (m) & Profundos & Someros \\
\hline Alta & $E-1$ & & & 2 & $1,6 \pm 0,2$ & 4 & \\
\hline Alta & $\mathrm{E}-2$ & & & & & 3 & 2 \\
\hline Alta & $E-3$ & 1 & $2,0 \pm 0,0$ & & & 3 & \\
\hline Alta & $E-4$ & 2 & $3,5 \pm 0,3$ & 2 & $1,8 \pm 0,6$ & 2 & 1 \\
\hline Media & $\mathrm{E}-5$ & & & 2 & $12,1 \pm 2,8$ & 3 & \\
\hline Media & $E-6$ & 2 & $1,8 \pm 0,7$ & 1 & $2,0 \pm 0,0$ & 2 & 2 \\
\hline Media & $E-7$ & 1 & $2,2 \pm 0,0$ & & & 3 & \\
\hline Media & $\mathrm{E}-8$ & & & & & 2 & 1 \\
\hline Media & $E-9$ & & & & & 1 & 1 \\
\hline Terminal & $E-10$ & & & & & & 1 \\
\hline Terminal & E-11 & & & & & & 1 \\
\hline Terminal & $\mathrm{E}-12$ & & & & & & \\
\hline
\end{tabular}

TABLA 4. Porcentaje de frecuencia y altura media de rápidos, saltos y cascadas presentes por zona fluvial. / Percentage of frequency and mean height for riffles, waterfall and cascade present for each fluvial zone.

\begin{tabular}{cccccc}
\hline \multirow{2}{*}{ Zona fluvial } & \multicolumn{2}{c}{ Saltos $(\mathrm{m})$} & \multicolumn{2}{c}{ Cascadas $(\mathrm{m})$} & Rápidos \\
\cline { 2 - 6 } & Frecuencia & Altura & Frecuencia & Altura & Frecuencia \\
\hline Alta & $50,0 \%$ & $1,75 \pm 0,3$ & $57,1 \%$ & $1,7 \pm 0,4$ & $51,7 \%$ \\
Media & $50,0 \%$ & $2,0 \pm 0,3$ & $42,9 \%$ & $7,0 \pm 7,1$ & $44,8 \%$ \\
Terminal & - & - & - & - & $3,4 \%$ \\
\hline
\end{tabular}

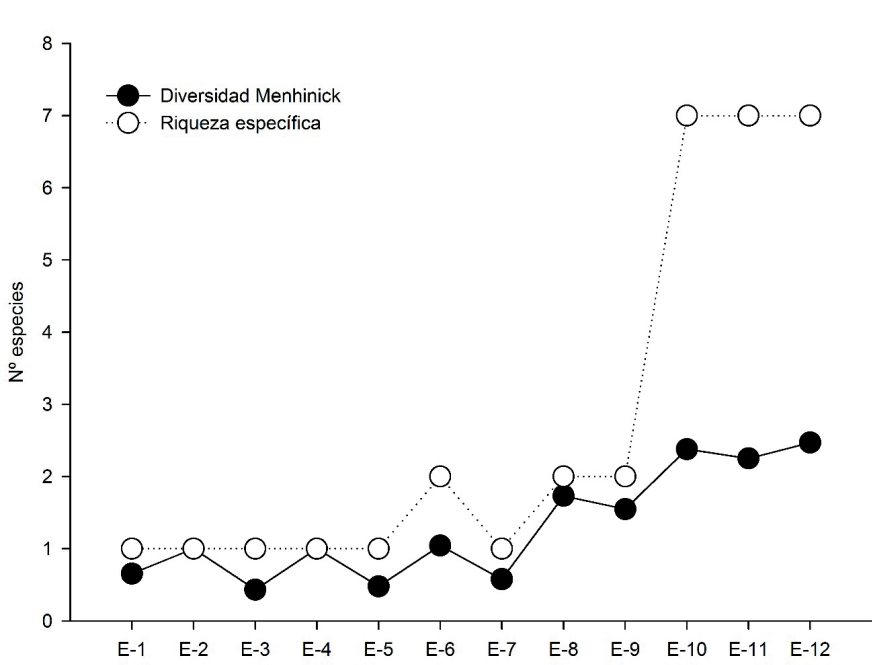

Figura 2. Riqueza específica y diversidad de Menhinick determinada para zona y estación de muestreo desarrollada en el río Quimán. / Specific richness and Menhinick diversity determined for area and sampling site, developed on the Quimán river.

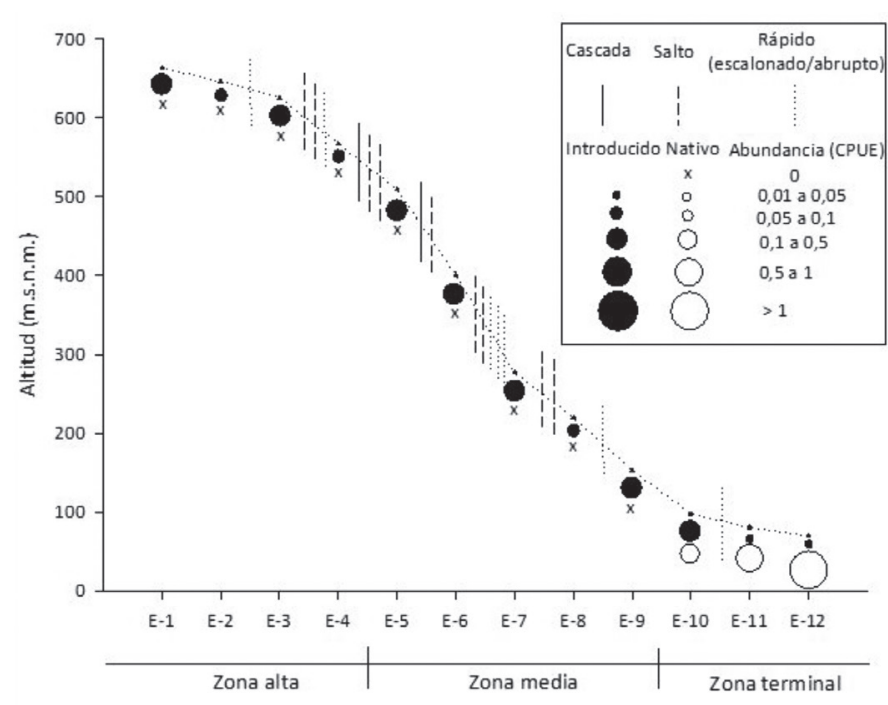

Figura 3. Comparación de CPUE entre nativos e introducidos, para todo el periodo de estudio entre las distintas zonas fluviales del río Quimán. / Comparison of CPUE between native and introduced, for the entire study period between the different river areas of the Quimán river. 


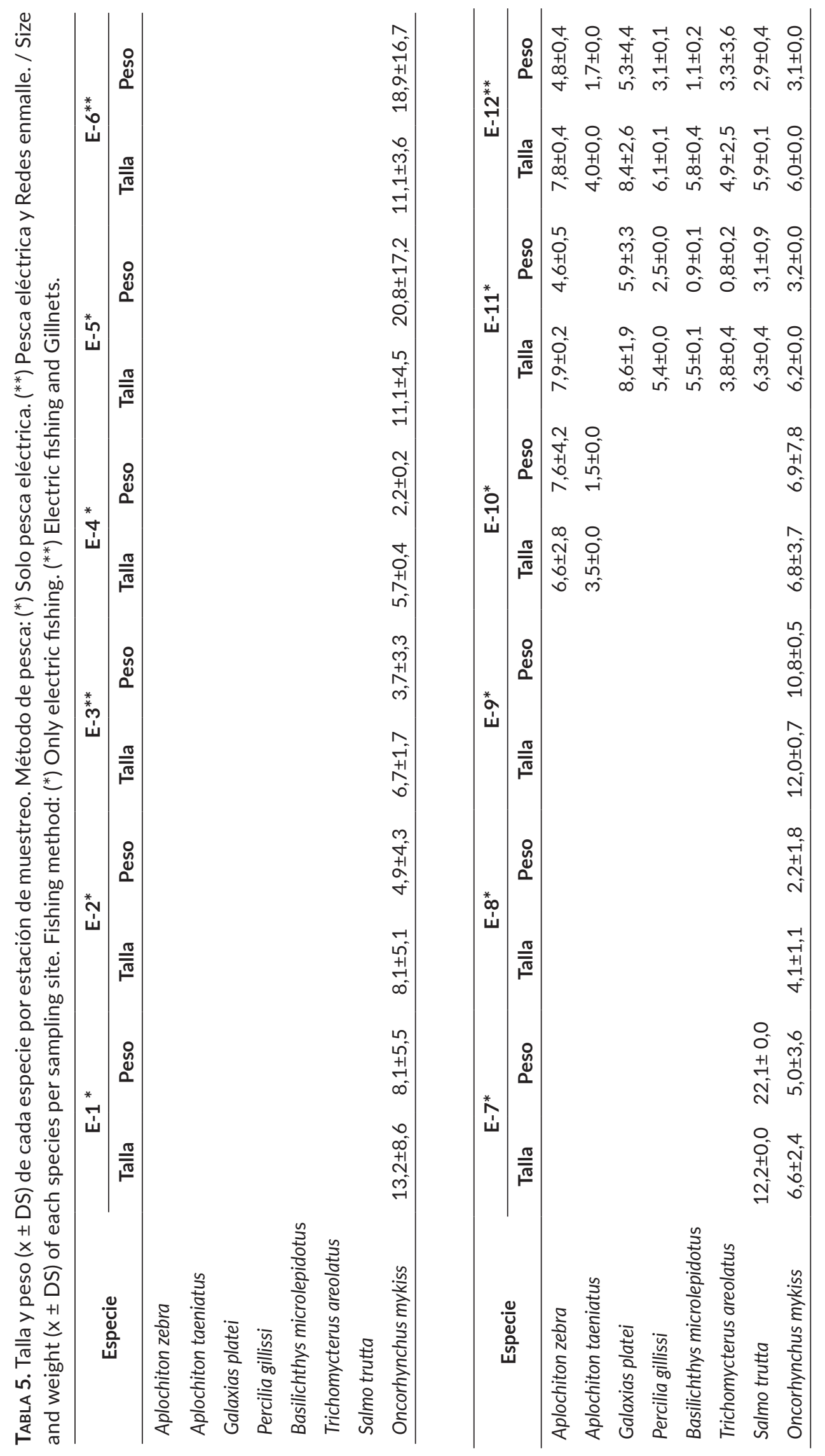




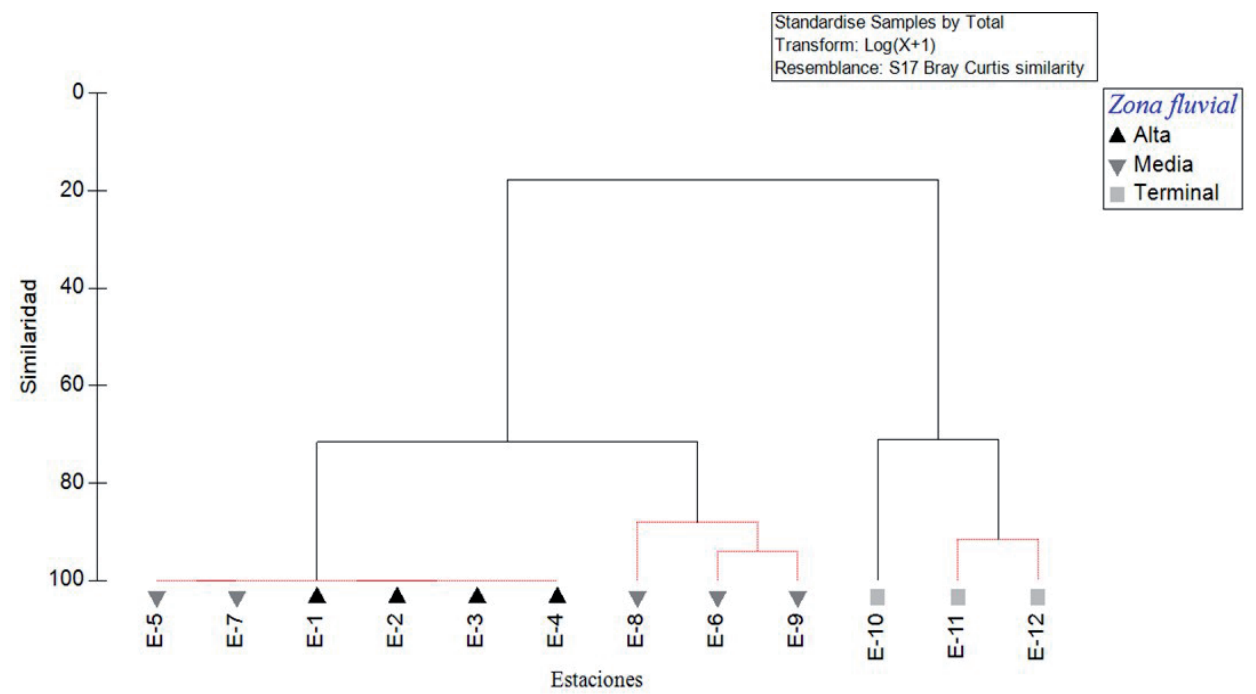

Figura 4. Clúster de similitud, basado en el índice de Bray-Curtis, para la distribución del ensamble de peces en el río Quimán. / Similarity cluster, based on the Bray-Curtis index, for the distribution of the fish assembly in the Quimán river.

\section{Patrones de disimiLitud de PeCES}

El análisis de NMDS (stress 0,1) y el clúster de Bray-Curtis revelaron tres grupos distintos con una similitud global de un $18 \%$ (Fig. 4). Dos grupos (zona alta y media) estuvieron compuestos solo por los salmonídeos Salmo trutta Linnaeus, 1758 y Oncorhynchus mykiss Walbaum, 1792 con un 60\% de similitud. El tercer grupo (zona terminal) representado por las especies: Aplochiton taeniatus Jenyns, 1842, A. zebra Jenyns, 1842, Galaxias platei Steindachner, 1898, Trichomycterus areolatus (Valenciennes 1848), Percilia gillissi (Girard 1855) y Basilichthys microlepidotus (Jenyns 1841) (Fig. 5). Un análisis de similitud ANOSIM global, corroboró diferencias significativas entre los grupos ( $r=0,62 \mathrm{p}=0,0006)$. Específicamente entre los ensambles de las zonas alta/terminal (ANOSIM, comparaciones pareadas $r=0,75 p=0,03$ ) y las zonas media/ terminal (ANOSIM, comparaciones pareadas, $r=0,68 p$ $=0,01)$. Del total de especies identificadas, el 75\% fueron encontradas exclusivamente en la zona terminal del Río Quimán. De acuerdo con el análisis SIMPER, los ensambles de la zona alta y media presentaron un bajo porcentaje de disimilitud (30\%), contribuida mayormente por O. mykiss $(96,7 \%)$ y S. trutta (3,3\%). Mientras que la diferencia entre la zona alta y terminal, exhibió una disimilitud del 83,4\%, contribuidas principalmente por las especies: T. areolatus (35,7\%), A. zebra (19,9\%), G. platei (16,9\%), O. mykiss (16,7\%), B. microlepidotus $(4,2 \%)$ y A. taeniatus $(2,2 \%)$ correspondientes al 95,8\%. Por último, la comparación entre las zonas media/ terminal mostraron una disimilitud del 81,7\%, aportadas principalmente por las especies: T. areolatus, A. zebra, G. platei, O. mykiss y B. microlepidotus con el $93,4 \%$ del total.
RELACIÓn ENTRE VARIABLES AMBIENTALES Y EL ENSAMBLE DE PECES El resultado del análisis BEST/BIOENV mostró a nivel global una baja relación entre las variables ambientales y la distribución del ensamble de peces ( $r=0,37 \mathrm{p}=0,03)$. La mayor correlación entre ambas matrices se presentó entre las variables velocidad de corriente $(r=0,37)$, pendiente $(r=$ $0,36)$ y la temperatura $(r=0,33)$.

Por otra parte, se determinó una preferencia de hábitat de bajas velocidades de corriente $\left(<0,64 \mathrm{~m} \mathrm{~s}^{-1}\right)$ y pendientes menores a un 3,5\% por parte de las especies nativas presentes en el Río Quimán (Fig. 5).

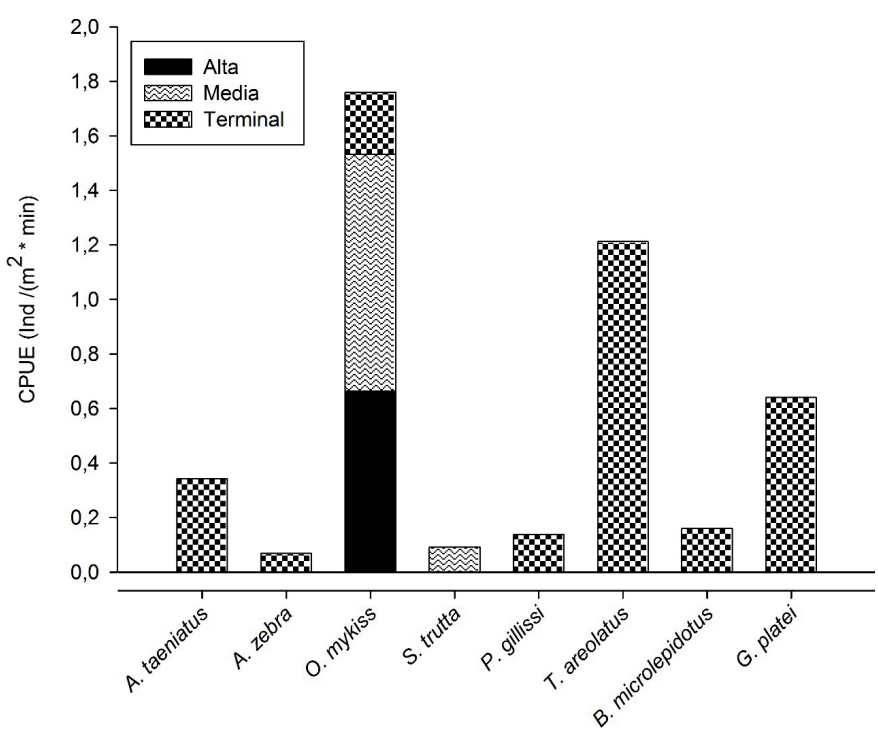

FIGURE 5. Comparación de abundancia (CPUE) entre las distintas zonas fluviales del río Quimán. / Comparison of abundance (CPUE) between the different river areas of the Quimán River. 


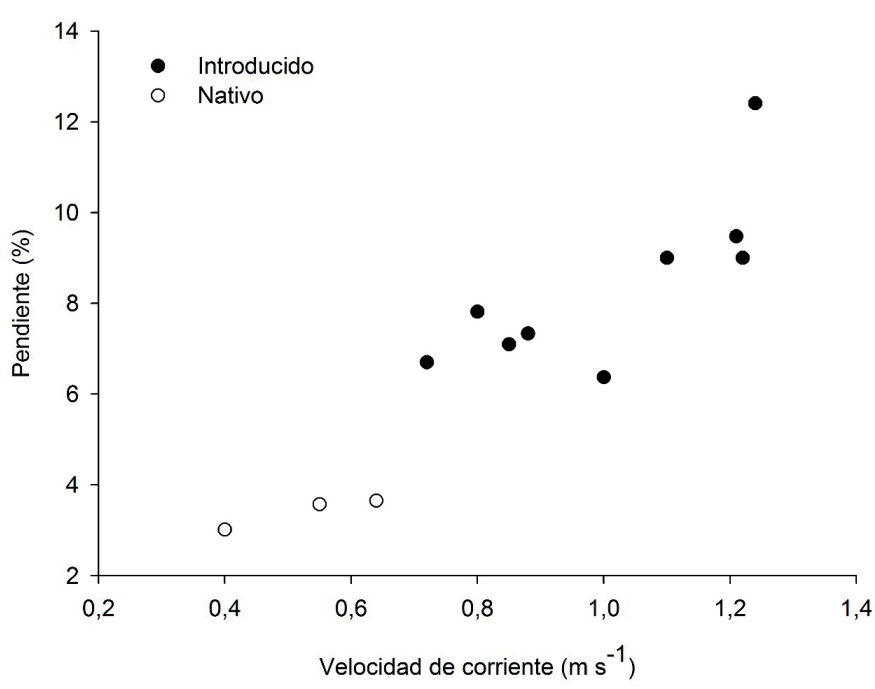

Figura 6. Correlación entre las variables geográficas velocidad de corriente $\left(\mathrm{m} \mathrm{s}^{-1}\right)$ y pendiente (\%), y su relación con la distribución espacial del ensamble de peces en el río Quimán. / Correlation between the geographic variables current velocity $\left(\mathrm{m} \mathrm{s}^{-1}\right)$ and slope (\%), and its relationship with the spatial distribution of the fish assembly in the Quimán river.

\section{DISCUSIÓN}

El ensamble íctico del Río Quimán estuvo constituido por 6 especies nativas (A. taeniatus, A. zebra, G. platei, T. areolatus, B. microlepidotus y $P$. gillissi) y 2 introducidas (O. mykiss y $S$. trutta). La composición de este ensamble presentó diferencias entre las distintas zonas del río, mostrando un aumento en la diversidad y riqueza de especies en el sentido de la corriente. Patrón longitudinal que ha sido registrado en otros ríos del país (Duarte et al. 1971; Habit 1998; Habit et al. 2007; Habit \& Victoriano 2012). Distribución espaciotemporal dependiente por una multiplicidad de factores bióticos (Vannote et al. 1980; Welcomme 1985; Growns et al. 2003) y abióticos (Oberdorff et al. 2001; Petry et al. 2003; Rahel 2007; Olden et al. 2010).

Las distintas zonas fluviales descritas en este trabajo mostraron diferencias significativas en las variables ambientales ORP y $\mathrm{pH}$, altitud, pendiente y velocidad de corriente. Estas últimas correlacionadas fuertemente entre sí: velocidad de corriente y altitud $(r=0,94)$, velocidad de corriente y pendiente $(r=0,91)$. Relación descrita frecuentemente en ríos de montaña (Lenzi et al. 2006) con una estructura sucesiva de hábitats de rápido-pozón (Comiti et al. 2007), variables geográficas que interfieren en las características físicas e hidráulicas de un hábitat (Clifford et al. 2006) e influyendo en el uso y ocupación de estos por parte de los peces (García et al. 2012).

Para el Río Quimán, el análisis BEST/BIOENV determinó una baja correlación entre las variables ambientales: velocidad de corriente $(r=0,37)$ y pendiente $(r=0,36)$ con la variación del ensamble íctico. Sin embargo, ambas variables son fundamentales para la restauración y composición de los ambientes fluviales (Rabeni \& Jacobson 1993) y un factor restrictivo en la ocupación y uso de hábitats (Walters et al. 2003; García et al. 2011, 2012). En los salmonídeos, la velocidad de corriente no parece ser una variable limitante para el uso y ocupación de hábitats de alta energía, debido a las adaptaciones y capacidades metabólicas, fisiológicas y natatorias (Gamperl et al. 2002; Jain \& Farrell 2003), pero la pendiente si actuaría como una eventual barrera restrictiva, afectando migraciones y desplazamientos activos de estas especies. Chisholm \& Hubert (1986) cuantificaron que pendientes sobre un $6,8 \%$ afectan negativamente la abundancia de salmonídeos, mientras que Kruse et al. (1997) describen la ausencia de poblaciones naturales en pendientes sobre el $10 \%$. No obstante, Steel \& Sheer (2003) aumentan este gradiente restrictivo, señalando que pendientes sobre un $20 \%$ son barreras naturales infranqueables para todo tipo de salmonídeos incluyendo los estadios adultos. Para los peces nativos, definidos por bajas tallas corporales $(<15 \mathrm{~cm}$ LT) y mayormente bentónicos y nocturnos (Vila et al. 1999; Link \& Habit 2015; Vila \& Habit 2015), ambas variables son taxativas, debido a que son especies adaptadas a ambientes someros, de baja velocidad de corriente (Arratia 1982, 1987; Campos et al. 1993a; Habit \& Victoriano 2005; Habit et al. 2007; García et al. 2011, 2012), con ciertas excepciones para los géneros Diplomystes y Trichomycterus que habitan ambientes de alta turbulencia y velocidad de corriente (Habit 2005; Scott et al. 2007; García et al. 2011; Habit \& Victoriano 2012). Esto concuerda con el patrón de distribución espacial que exhibieron las especies nativas en el río, distribuidas solo en la zona terminal, cercana a la desembocadura del Lago Ranco, en donde las velocidades de corriente y pendiente son menores. Mientras que los salmonídeos mostraron un uso extensivo de las distintas zonas del Río Quimán.

Por otra parte, se ha postulado que la ausencia de peces nativos en el ritrón de las cuencas se debe a los efectos históricos recientes de glaciación (Pleistoceno tardío) que pudieron haber impedido la colonización por parte de especies nativas (Campos et al. 1993; Cussac et al. 2004). Mientras que otros autores postulan a cambios orográficos relacionados con el levantamiento temprano de la Cordillera de Los Andes (hace 1,5 millones de años) que ocasionó alteraciones en la dirección del flujo, retrogresiones y trasvasijes de las cuencas alto andinas desde la vertiente oriental hacia la vertiente occidental de la cordillera, 
permitiendo la colonización y distribución de algunas especies nativas (Galáxidos) hacia zonas costeras del Océano Pacífico y zonas secundarias al zona norte de la Patagonia Chilena. Colonizando desde las líneas costeras y paleolagos hacia tramos superiores de las cuencas (Zemlak et al. 2008, 2010, 2011; Habit et al. 2012; Vera-Escalona et al. 2015; Cussac et al. 2016; Vera-Escalona et al. 2019).

Esta ausencia de ictiofauna nativa en los tramos superiores de los ríos se debería a desplazamientos forzados producto de la ocupación y colonización de salmonídeos, generando efectos negativos para las especies nativas (Habit et al. 2003, 2007), especialmente sobre los galáxidos (Macchi et al. 1999, 2007; McDowall 2006) modificando sus patrones de ocupación y distribución espacial (Habit et al. 2015), efectos sobre la abundancia (Arismendi et al. 2009; Correa \& Hendry 2012; Habit et al. 2010), diversidad y composición genética (Vera-Escalona et al. 2019), interacciones tróficas (Macchi et al. 1999, 2007; Elgueta et al. 2013; ), tasas de crecimiento (Young et al. 2010) y uso de hábitats (Vargas et al. 2010).

Respecto a los saltos y cascadas, Robinson \& Rand (2005) las definen como límites de discontinuidad para las comunidades de peces, que evitan sus movimientos hacia tramos superiores. Además, se ha documentado que incrementan la segregación de los hábitats y fragmentan las poblaciones, aumentando la diversidad genética (Diener et al. 2007; Rahel 2007; Olden et al. 2010), aunque los cambios orográficos y climáticos, durante el periodo Cuaternario en la Patagonia, actuaron como barreras físicas impermeables que afectaron la diversidad genética de especies nativas en poblaciones cerradas, mediante el aislamiento y segregación en zonas de refugios (Zemlak et al. 2008, 2010, 2011; VeraEscalona et al. 2015, 2018). Sin embargo, el mecanismo de aislamiento o "landlocking" ha sido reconocido como un importante precursor de diversificación a nivel poblacional y especie (Allibone \& Wallis 1993; Waters \& Wallis 2001). En cambio, pequeñas barreras como los rápidos, al ser más permeables actuarían como un filtro ecológico, generando diferencias en la composición y estructura de los ensambles ícticos (Silva et al. 2015). No obstante, la eliminación de cualquiera de estas barreras provocaría un proceso de homologación de los ensambles, afectando la diversidad y pérdida de hábitats (Júlio Júnior et al. 2009). En el Río Quimán la presencia de estas barreras geográficas presentaron una mayor frecuencia en los tramos alto y medio en el río, con alturas que fluctuaron entre 2,5 a 14 metros. Alturas de barreras que son consideradas infranqueables, según lo establecido por Meixler et al. (2009) quienes determinaron mediante un modelo predictivo que barreras sobre 6 metros de altura bloquean cualquier migración o flujo hacia tramos superiores para peces migratorios. Sin embargo, la presencia de salmonídeos sobre estas barreras en el río, se debería a liberaciones de carácter espontáneas por parte de pescadores locales, desarrolladas durante la década de 1990, con el objetivo de generar un área de recreación de pesca deportiva, según lo relatado por los mismos lugareños. Actividad que se ha realizado a través del tiempo en los distintos sistemas fluviales y lacustres en el país (Basulto 2003) y a nivel global (Welcomme 1988).

Por otra parte, la presencia de una piscicultura y una planta de tratamiento de agua servida en la zona terminal del río, aparentemente no han provocado efectos negativos sobre la ictiofauna, tanto en su riqueza de especies como en su diversidad. Por el contrario, ambos parámetros en este tramo del río han aumentado mostrando una tendencia opuesta a lo reportado por Habit et al. (2005) y Aedo et al. (2009) quienes describieron una afectación negativa en la diversidad de peces nativos, o más aún, la ausencia de peces (Dyer et al. 2003), producto del enriquecimiento de materia orgánica del agua debido a la descarga de aguas servidas. Por otra parte, Parra et al. (2003), en sistemas lóticos con descargas de aguas servidas, reportaron cambios en la estructura y composición comunitaria, dominada por especies introducidas tolerantes como la carpa común (Cyprinus carpio Linneo, 1758) y pejerrey argentino (Odontesthes bonariensis Valenciennes, 1835). En cuanto a la amenaza de potenciales escapes de salmonídeos desde la piscicultura, esto puede traer consigo interferencias negativas graves sobre las poblaciones de peces nativos, pero de acuerdo a los resultados no se evidencian en este último tramo del río, durante el periodo de estudio.

Respecto a las centrales hidroeléctricas de paso, estas también generan una modificación y alteración en el ecosistema fluvial original, cambiando el régimen del río y transformando el ambiente fluvial con repercusiones en la biodiversidad, abundancia, zonas de postura y alimentación de peces y la perdida de diversidad genética, pudiendo afectar la capacidad de adaptación de las especies a futuros cambios climáticos y ambientales del entorno (Habit et al. 2007a; Renöfält et al. 2010; García et al. 2011; Benejam et al. 2016; Vera-Escalona et al. 2018).

En cuanto a los usos de hábitats por parte de las especies nativas, la mayoría fueron encontradas en zonas someras de baja velocidad de corriente, relacionadas a las riberas y litoral lacustre. El género Aplochiton fue capturado en rápidos someros asociados a las riberas y desembocadura del río, junto con las especies $P$. gillissi y $T$. areolatus, concordando con lo reportado en otros estudios (Campos 1969, 1984; García et al. 2011, 2012). Mientras G. platei, especie típica de ambientes profundos de sistemas lacustres (Habit et 
al. 2010, 2012, Sobenes et al. 2013) y fluviales (Milano 2006), fue identificada en pozas profundas, cercanas a la desembocadura. B. microlepidotus, especie abundante en estuarios, desembocaduras de ríos y lagos (Comte \& Vila 1992; García et al. 2012; Laborde et al. 2016) fue capturada en el litoral lacustre.

Los patrones de ocupación y distribución espacial de los peces nativos en el Río Quimán estarían relacionados con las variables geográficas, específicamente por la presencia de barreras naturales infranqueables para los peces migratorios (> $6 \mathrm{~m}$ de altura), describiendo una variación espacial en la composición del ensamble íctico a lo largo de los distintos tramos del río. En los tramos superiores del río solo constituidos por salmonídeos y el tramo terminal dominados por especies nativas, con un uso preferencial de ambientes de baja velocidad de corriente $\left(<0,64 \mathrm{~m} \mathrm{~s}^{-1}\right)$ y pendientes menores al 3,5\%. La presencia de salmonídeos sobre estas barreras infranqueables se explica por acciones de liberación antrópica de carácter espontaneas por parte de pescadores locales.

Se establece la necesidad de recabar más información para determinar los efectos que produce la orografía (barreras geográficas) sobre la riqueza y diversidad de peces nativos, considerando sus pequeños tamaños corporales $(<$ $15 \mathrm{~cm} \mathrm{LT)}$ ), capacidad motora, anatomía y preferencias de uso de hábitats someros de baja velocidad de corriente.

\section{AGRADECIMIENTOS}

Agradecemos al personal de la Hidroeléctrica Las Flores por la logística y los accesos, a Reinaldo Rivera por sus comentarios y sugerencias, y a Francisco "Pancho" Rivera por su guía y apoyo en Arc Gis.

\section{REFERENCIAS}

Aedo, J., Belk, M., Habit, E. 2009. Geographic variation in age, growth and size of Percilia irwini from south-central Chile. Journal of Fish Biology 74(1): 278-284.

Allibone, R.M., Wallis, G.P. 1993. Genetic variation and diadromy in some native New Zealand galaxiids (Teleostei: Galaxiidae). Biological Journal of the Linnean Society 50(1): 19-33.

Araujo-Lima, C., Portugal, L., Ferreira, E. 1986. Fish-macrophyte relationship in Anavilhanas Archipelago, a black water system in the central Amazon. Journal of Fish Biology 29: 1-11.

Arismendi, I., Nahuelhual, L. 2007. Non-native Salmon and
Trout Recreational Fishing in Lake Llanquihue, Southern Chile: Economic Benefits and Management Implications. Reviews in Fisheries Science 15(4): 311-325.

Arratia, G. 1982. Peces del altiplano de Chile. En: Veloso, A., Bustos, L. (Eds.) El Hombre y los ecosistemas de montaña: 93-133. Ediciones Oficina Regional de Ciencia y Tecnología de la UNESCO para América Latina y el Caribe. Montevideo, Uruguay.

Arratia, G. 1987. Description of the primitive family Diplomystidae (Siluriformes, Teleostei, Pisces): morphology, taxonomy and phylogenetic implications. Bonn Zoological Monographs 24: 1-20.

Arratia, G.1992. Development and variation of the suspensorium of primitive catfishes (Teleostei: Ostariophysi) and their phylogenetic relationships. Bonner zoologische Monographien 32: 1-149.

Basulto, S. 2003. El largo viaje de los salmones: una crónica olvidada, propagación y cultivo de especies acuáticas en Chile, Editorial Maval, Santiago de Chile. 229 pp.

Beecher, H., Dott, E., Fernav, R. 1988. Fish species richness and stream order in Washington state stream. Environmental Biology of Fishes 22(3): 193-209.

Benejam, L., Saura-Mas, S., Bardina, M., Solà, C., Munné, A., García-Berthou, E. 2016. Ecological impacts of small hydropower plants on headwater streams fish: from individual to community effects. Ecology of Freshwater Fish 25: 295-306.

Campos, H. 1969. Reproducción del Aplochiton taeniatus Jenyns. Boletín del Museo Nacional de Historia Natural. Tomo XXIX: 207-222.

Campos, H. 1984. Productividad íctica de ríos y lagos araucanos. En: Vila, I, Fagetti, E. (Eds.) Trabajos presentados al Taller Internacional sobre ecología y manejo de peces en lagos y embalses. Santiago, Chile, 5-10 de noviembre de 1984. COPESCAL Documento Técnico (4). 237 pp.

Campos, H., Alay, F., Ruíz, V., Gavilán, J. 1993. Antecedentes biológicos de la fauna íctica presente en la hoya hidrográfica del río Biobío. In: Faranda F., Parra O. (Eds.) Evaluación de la calidad del agua y ecología del sistema limnético y fluvial del río Biobío: 249-278. Serie Monografías Científicas EULA, Concepción, Chile.

Chisholm, I., Hubert, W. 1986. Influence of stream gradient on standing stock of brook trout in the snowy range, Wyoming. Northwest Science 60(2): 137-139.

Clarke, K. 1993. Non-parametric multivariate analyses of changes in community structure. Australian Journal of Ecology 18: 117-143.

Clarke, K.R., Gorley, R.N. 2005. PRIMER v.6: User Manual/ Tutorial. PRIMER-E Ltda., Playmouth, UK. Available at: https://www.primer-e.com/. 
Clarke, K.R., Warwick, R.M., Somerfield, P.J., Gorley, R.N. 2005. Change in marine communities: an approach to statistical analysis and interpretation. $3^{\text {rd }}$. PRIMER-E Ltda, Plymouth, UK.

Clifford, N., Harmar, O., Harvey G., Petts G. 2006. Physical habitat, eco-hydraulics and river design: a review and re-evaluation of some popular concepts and methods. Aquatic Conservation: Marine and Freshwater Ecosystems 16(4): 389-408.

Colin, N., Piedra, P., Habit, E. 2012. Variaciones espaciales y temporales de las comunidades ribereñas de peces en un sistema fluvial no intervenido: Río San Pedro, Cuenca del Río Valdivia (Chile). Gayana 76(supl. 1): 24-35.

Comiti, F., Mao, L., Wilcox, A., Wohl, E., Lenzi, M. 2007. Fieldderived relationships for flow velocity and resistance in high-gradient streams. Journal of Hydrology 340(1-2): 48-62.

Comte, S., Vila, I. 1992. Spawning of Basilichthys microlepidotus (Jenyns). Journal of Fish Biology 41: 971-981.

Correa, C., Hendry, A. 2012. Invasive salmonids and lake order interact in the decline of puye grande Galaxias platei in western Patagonia lakes. Ecological Applications 22(3): 828-842.

Cussac, V., Ortubay, S., Iglesias, G., Milano, D., Lattuca, M.E., Barriga, J.P., Battini, M., Gross, M. 2004. The distribution of South American galaxiid fishes: the role of biological traits and post-glacial history. Journal of Biogeography 31: 103-121.

Cussac, V., Habit, E., Ciancio, J., Battini, M., Rossi, C., Barriga, J.P., Baigún, C., Crichigno, S. 2016. Freshwater fishes of Patagonia: conservation and fisheries. Journal of Fish Biology 89(1): 1068-1097.

Dyer, B. 2000. Systematic review and biogeography of the freshwater fishes of Chile. Estudios Oceanológicos 19: 77-98.

Dyer, S., Peng, C., Mcavoy, D., Fendinger, N., Masscheleyn, P., Castillo, L., Lim, J. 2003. The influence of untreated wastewater to aquatic communities in the Balatuin River, The Philippines. Chemosphere. 52(1): 43-53.

Eigenmann, C. 1927. The freshwater fishes of Chile. Memories of the National Academy of Sciences 22: 1-63.

Elgueta, A., González, J., Ruzzante, D., Walde, S., Habit, E. 2013. Trophic interference by Salmo trutta on Aplochiton zebra and Aplochiton taeniatus in southern Patagonian lakes. Journal of Fish Biology 82: 430-443.

Gamperl, A., Rodnick, K., Faust, H., Venn, E., Bennett, M., Crawshaw, L., Keeley, E., Powell, M., Li, H. 2002. Metabolism, swimming performance, and tissue biochemistry of high desert redband trout (Oncorhynchus mykiss): evidence for phenotypic differences in physiological function. Physiological and Biochemical Zoology 75(5): 413-431.

García, A., Jorde, K., Habit, E., Caamaño, D., Parra, O. 2011. Downstream environmental effects of dam operations: changes in habitat quality for native fish species. River Research and Applications 27(3): 312-327.

García, A., González, J., Habit, E. 2012. Caracterización del hábitat de peces nativos en el río San Pedro (cuenca del río Valdivia, Chile). Gayana 76(supl. 1): 36-44.

Growns, I., Gehrke, P., Astles, L., Pollard, D. 2003. A comparison of fish assemblages associated with different riparian vegetation types in the Hawkesbury-Nepean River system. Fisheries Management and Ecology 10: 209220.

Habit, E., Victoriano, P., Ruiz, A. 2003. Variaciones espaciotemporales del ensamble de peces de un sistema fluvial de bajo orden del centro-sur de Chile. Revista Chilena de Historia Natural 76: 3-14.

Habit, E., Parra, O., Valdovinos, C. 2005. Ictiofauna de un sistema fluvial receptor de aguas servidas: Respuestas a una nueva planta de tratamiento (Rio Quilque, Chile central). Gayana 69(1): 94-103.

Habit, E., Belk, M., Victoriano, P., Jaque, E. 2007. Spatiotemporal distribution patterns and conservation of fish assemblages in a Chilean coastal river. Biodiversity and Conservation 16: 3179-3191.

Habit, E., Belk, M., Parra, O. 2007. Response of the riverine fish community to the construction and operation of a diversion hydropower plant in central Chile. Aquatic Conservation: Marine and Freshwater Ecosystems 17(1): 37-49.

Habit, E., Piedra, P., Ruzzante, D., Walde, S., Belk, M., Cussac, V., González, J., Colin, N. 2010. Changes in the distribution of native fishes in response to introduced species and other anthropogenic effects. Global Ecology and Biogeography 19(5): 697-710.

Habit, E., González, J., Ruzzante, D., Walde, S. 2012. Native and introduced fish species richness in Chilean Patagonian lakes: inferences on invasion mechanisms using salmonid-free lakes. Diversity and Distributions 18: 1153-1165.

Habit, E., González, J., Ortiz-Sandoval, J., Elgueta, A., Sobenes, C. 2015. Efectos de la invasión de salmónidos en ríos y lagos de Chile. Ecosistemas 24(1): 43-51.

Hawkins, D., Quinn, T. 1996. Critical swimming velocity and associated morphology of juvenile coastal cutthroat trout (Oncorhynchus clarki clarki), steelhead trout (Oncorhynchus mykiss) and their hybrids. Canadian Journal of Fisheries and Aquatic Sciences 53(7): 14871496. 
Hubert, N., Renno, J. 2006. Historical biogeography of South American freshwater fishes. Journal of Biogeography 33: 1414-1436.

Huechan, A.M. 1997. Análisis de subcuencas en torno al Lago Ranco, Provincia de Valdivia, Región de Los Lagos. Tesis para optar al título de profesora de Historia, Geografía y Educación Cívica, Facultad de Filosofía y Humanidades, Universidad Austral de Chile.

Júlio Júnior, H., Deitós, C., Agostinho, A., Simone-Pavanelli, C. 2009. A massive invasion of fish species after eliminating a natural barrier in the upper Paraná River basin. Neotropical Ichthyology 7(4): 709-718.

Krebs, C.J. 1989. Ecological methodology. Harper Collins, New York. 459 pp.

Kruse, C.G., Hubert, W.A., Rahel, F.J. 1997. Geomorphic influences on the distribution of Yellowstone cutthroat trout in the Absaroka Mountains, Wyomming. Transactions of the American Fisheries Society 126: 418-427.

Laborde, A., González, A., Sanhueza, C., Arriagada, P., Wilkes, M., Habit, E., Link, O. 2016. Hydropower development, riverine connectivity, and non-sport fish species: criteria for hydraulic design of fishways. River Research and Applications 32(9): 1949-1957.

Lenzi, M., Mao, L., Comiti, F. 2006. Effective discharge for sediment transport in a mountain river: computational approaches and geomorphic effectiveness. Journal of Hydrology 326: 257-276.

Link, O., Habit, E. 2015. Requirements and boundary conditions for fish passes of non-sport fish species based on Chilean experiences. Reviews in Environmental Science and Biotechnology 14: 9-21.

Lotrich, V. 1973. Growth, production and community composition of fishes inhabiting a first, second, and third order stream of eastern Kentucky. Ecological Monographs 43: 377-397.

Jain, K., Farrel A. 2003. Influence of seasonal temperature on the repeat swimming performance of rainbow trout Oncorhynchus mykiss. Journal of Experimental Biology 206(20): 3569-3579.

Macías-Zamora, R., Vidaurri-Sotelo, A., Santana-Hernández, H. 1994. Análisis de la tendencia de captura por unidad de esfuerzo en la pesquería del pez vela en el Pacífico Mexicano. Ciencias Marinas 20(3): 393-408.

Macchi, P., Cussac, V.E., Alonso, M., Denegri, M. 1999. Predation relationships between introduced salmonids and the native fish fauna in lakes and reservoirs in the northern Patagonia. Ecology of Freshwater Fish 8(4): 227-236.

Macchi, P., Pascual, M., Vigliano, P. 2007. Differential piscivory of the native Percichthys trucha and exotic salmonids upon the native forage fish Galaxias maculatus in Patagonian
Andean lakes. Limnologica 37: 76-87.

McDowall, R. 2006. Crying Wolf, crying foul, or crying shame: alien salmonids and a biodiversity crisis in the southern cool-temperate galaxioid fishes?. Reviews in Fish Biology and Fisheries 16: 233-422.

Meixler, M., Bain, M., Walter, T. 2009. Predicting barrier passage and hábitat suitability for migratory fish species. Ecological Modelling 220: 2782-2791.

Milano, D., Ruzzante, V., Cussac, P., Macchi, R., Ferriz, J., Barriga, J., Aigo, J., Lattuca, M., Walde, S. 2006. Latitudinal and ecological correlates of morphological variation in Galaxias platei (Pisces, Galaxiidae) in Patagonia. Biological Journal of the Linnean Society 87: 69-82.

Oberdorff, T., Pont, D., Hugueny, B., Chessel, D. 2001. A probabilistic model characterizing fish assemblages of French rivers: a framework for environmental assessment. Freshwater Biology 46: 399-415.

Olden, J., Kennard, M., Leprieur, F., Tedesco, P., Winemiller, K., García-Berthou, E. 2010. Conservation biogeography on freshwater fishes: recent progress and future challenges. Diversity and Distributions 16(3): 496-513.

Parot, P. 2012. Estudio Hidrológico Río Quimán XIV Región (Informe Técnico). Proyecto Ampliación Minicentral Hidroeléctrica Las Flores. Valdivia, Chile. 57 pp.

Parra, O., Valdovinos, C., Urrutia, R., Cisternas, M., Habit, E., Mardones, M. 2003. Caracterización y tendencias tróficas de cinco lagos costeros de Chile central. Limnética 22(1-2): 51-83.

Petry, A., Agostinho, A., Gomes, L. 2003. Fish assemblages of tropical floodplain lagoons: exploring the role of connectivity in a dry year. Neotropical Ichthyology 1(2): 111-119.

Piedra, P., Habit, E., Oyanedel, A., Colin, N., Solis-Kufí, K., González, J., Jara, A., Ortiz, N., Cifuentes, R. 2012. Patrones de desplazamiento de peces nativos en el Río San Pedro (Cuenca del Río Valdivia, Chile). Gayana 76(supl. 1): 59-70.

Pusey, B., Kennard, M. 1996. Species richness and geographical variation in assemblage structure of the freshwater fish fauna of the wet tropics region of northern Queensland. Marine and Freshwater Research 47: 563-573.

Rabeni, C., Jacobson, R. 1993. The importance of fluvial hydraulics to fish-habitat restoration in low-gradient alluvial streams. Freshwater Biology 29(2): 211-220.

Rahel, F. 2007. Biogeographic barriers, connectivity and homogenization of freshwater faunas: it's a small world after all. Freshwater Biology 52: 696-710.

Ramírez, A. 1999. Ecología aplicada: Diseño y análisis estadístico. Fundación Universitaria Jorge Tadeo Lozano, Bogota. $325 \mathrm{pp}$. 
Renöfält, B.E., Jansson, R., Nilsson, C. 2010. Effects of the hydropower generation and opportunities for environmental flow management in Swedish riverine ecosystems. Freshwater Biology 55(1): 49-67.

Ricklefs, R.E., Schluter, D. 1993. Species diversity: regional and historical influences. In: Ricklefs, R.E., Schluter, D. (Eds). Species Diversity in Ecological Communities: Historical and Geographical Perspectives: 350-363. The University of Chicago Press, Chicago, IL, USA.

Robinson, J., Rand, P. 2005. Discontinuity in fish assemblages across an elevation gradient in a southern Appalachian watershed, USA. Ecology of Freshwater Fish 14: 14-23.

Sheer, M., Steel, E. 2006. Lost watersheds: barriers, aquatic habitat connectivity, and salmon persistence in the Willamette and lower Columbia River basins. Transactions of the American Fisheries Society 135: 1654-1669.

Silva, J., Gubiani, E., Piana, P., Delariva, R. 2015. Effects of small natural barrier on the spatial distribution on the fish assemblage in the Verde River, upper Paraná River basin, Brazil. Brazilian Journal of Biology 76(4): 851-863.

Torrente-Vilara, G., Zuanon, J., Leprieur, F., Oberdorff, T., Tedesco, P. 2011. Effects of natural rapids and waterfalls on fish assemblage structure in the Madeira River (Amazon Basin). Ecology of Freshwater Fish 20: 588-597.

Vannote, R., Wayne, G., Cummins, K., Sedell, J., Cushing, C. 1980. The river continuum concept. Canadian Journal of Fisheries and Aquatic Sciences 37: 130-137.

Vargas, P., Arismendi, I., Lara, G., Millar, J. \& Peredo, S. 2010. Evidence of microhabitat overlap between juvenile of introduced salmon Oncorhynchus tshawytscha and the native fish Trichomycterus areolatus in the Allipén River, Chile. Revista de Biología Marina y Oceanografía 45: 285-292.

Vera-Escalona, I., Habit, E., Ruzzante, D.E. 2015. Echoes of a distant time: Effects of historical processes on contemporary genetic patterns in Galaxias platei in Patagonia. Molecular ecology 24(16): 4112-4128.

Vera-Escalona, I., Senthivasan, S., Habit, E., Ruzzante, D.E. 2018. Past, present, and future of a freshwater fish metapopulation in a threatened landscape. Conservation biology 32(4): 849-859.

Vera-Escalona, I., Habit, E., Ruzzante, D.E. 2019. Invasive species and postglacial colonization: their effects on the genetic diversity of a Patagonian fish. Proceedings of the Royal Society B 286(1897): 20182567.

Vila, I., Fuentes, L., Contreras, M. 1999. Peces Límnicos de Chile. Boletín del Museo Nacional de Historia Natural, Chile 48: 61-75.

Vila, I., Habit, E. 2015. Current situation of the fish fauna in the Mediterranean region of Andean river systems in Chile. FISHMED Fishes in Mediterranean Environments 002: 19.

Walters, D., Leigh, D., Freeman, C., Freeman, B., Pringle, C. 2003. Geomorphology and fish assemblages in a piedmont river basin, U.S.A. Freshwater Biology 48: 1950-1970.

Waters, J.M., Wallis, G.P. 2001. Cladogenesis and loss of the marine life-history phase in freshwater galaxiid fishes (Osmeriformes: Galaxiidae). Evolution 55(3): 587-597.

Welcomme, R. 1985. River fisheries. FAO Fisheries Technical Paper 262: 1-318.

Welcomme, R. 1988. International introductions of inland aquatic species. FAO Fisheries Technical Paper 294. Food and Agriculture Organization of the United Nations, Rome, Italia.

Young, K., Dunham, J., Sthephenson, J., Terreau, A., Thailly, A., Gajardo, G., García de Leaniz, C. 2010. A trial of two trouts: comparing the impacts of rainbow and brown trout on a native galaxiid. Animal Conservation 13: 399 410.

Zemlak, T.S., Habit, E., Walde, S., Battini, M., Adams, E., Ruzzante, D.E. 2008. Across the southern Andes on fin: glacial refugia, drainage reversals and a secondary contact zone revealed by the phylogeographical signal of Galaxias platei in Patagonia. Molecular Ecology 17(23): 5049-5061.

Zemlak, T.S., Habit, E., Walde, S., Correa, C., Ruzzante, D.E. 2010. Surviving historical Patagonian landscapes and climate: molecular insights from Galaxias maculatus. BMC Evolutionary Biology 10(1): 67.

Zemlak, T.S., Walde, S., Habit, E., Ruzzante, D.E. 2011. Climateinduced changes to the ancestral population size of two Patagonian galaxiids: the influence of glacial cycling. Molecular Ecology 20(24): 5280-5294.

Zeug, S., Winemiller, K. 2008. Relationships between hydrology, spatial heterogeneity, and fish recruitment dynamics in a temperate floodplain river. River Research and Applications 24: 90-102.

Received: 16.05 .2018

Accepted: 31.07.2020 